Pacific Journal of Mathematics

THE ABSOLUTE GALOIS GROUP OF A PSEUDO REAL 


\title{
THE ABSOLUTE GALOIS GROUP OF A PSEUDO REAL CLOSED ALGEBRAIC FIELD
}

\author{
DAN HARAN AND MOSHE JARDEN
}

\begin{abstract}
The absolute Galois group of a PRC (= pseudo real closed) field is characterized as a real projective group. Specifically, it is known that if $E$ is a PRC field, then its absolute Galois group $G(E)$ is real projective. Conversely, if $G$ is a real projective group, then there exists a PRC field $E$ such that $G(E) \cong G$. The construction of $E$ makes it of infinite transcendence degree over $\mathbf{Q}$. However, if a field $E$ is algebraic over $\mathbf{Q}$, then rank $G(E) \leq \boldsymbol{\aleph}_{0}$. Therefore it is natural to ask whether for a given real projective group $G$ of rank $\leq \boldsymbol{\aleph}_{0}$ we may choose $E$ to be algebraic over Q.

There are two reasons for asking this question. First of all, the corresponding question for projective groups and PAC fields is known to have an affirmative answer, since there exist algebraic PAC fields $E$ such that $G(E) \cong \hat{F}_{\omega}=$ the free profinite group of ranks $\kappa_{0}$ and since every projective group $G$ of rank $\leq \aleph_{0}$ is isomorphic to a closed subgroup of $\hat{F}_{\omega}$. A generalization of this fact to real projective groups and PRC fields will be a contribution to the desired description of the closed subgroups of $G(\mathrm{Q})$. Secondly, an affirmative answer to this question will give us a necessary tool to the study of the elementary theory of all PRC fields which are algebraic over $Q$.

The main goal of this work is indeed to give the desired affirmative answer:
\end{abstract}

THEOREM. If $K$ is a countable formally real Hilbertian field and $G$ is a real projective group of rank $\leq \aleph_{0}$, then there exists a PRC algebraic extension $E$ of $K$ such that $G(K) \cong G$.

In order to make this introduction self-contained we repeat the basic definitions involved in the Theorem.

A field $E$ is said to be PRC (= pseudo real closed), if every absolutely irreducible variety $V$ defined over $K$, which has a simple $\bar{K}$-rational point in every real closed field $\bar{K}$ containing $K$, has a $K$-rational point.

A diagram

$$
\begin{aligned}
& G \\
& \downarrow \varphi \\
\rightarrow & A
\end{aligned}
$$


of epimorphisms of profinite groups is said to be a real embedding problem for $G$ if for every involution (i.e. element of order 2) $g$ of $G$ such that $\varphi(g) \neq 1$ there exists an involution $b$ of $B$ such that $\alpha(b)=\varphi(g)$. The problem is finite if $B$ is a finite group.

A profinite group is said to be real projective if the subset Inv $\mathrm{G}$ of all involutions of $G$ is closed and every finite real embedding problem (1) for $G$ is solvable, i.e., there exists a homomorphism $\gamma: G \rightarrow B$ such that $\alpha \circ \gamma=\varphi$.

We sketch here the basic ideas involved in the proof of the Theorem. We choose a closed system $X$ of representatives for the conjugacy classes of Inv $\mathrm{G}$ and choose a sequence $S$ of generators for $G$ that converges to 1 . Let $\hat{D}=D(X, S)$ be the real free group in the sense of [6] with the basis $(X, S)$. Then the obvious surjection $\hat{D} \rightarrow G$ induces a cover of the corresponding Artin-Schreier structures [5]. Hence it has a section, and consequently $G$ is isomorphic to a closed subgroup of $\hat{D}$.

Secondly, we show that the Boolean space $X_{\omega}=\{ \pm 1\}^{\mathbf{N}}$ together with a discrete sequence form a basis for a universal real free group $\hat{D}_{\omega}$ of countable rank, which, among other properties, contains all real free groups of rank $\leq \boldsymbol{\aleph}_{0}$ as closed subgroups.

Using a theorem of Binz-Neukirch-Wenzel about open subgroups of free product of profinite groups and a method of Lubotzky-v.d. Dries, we embed $\hat{D}_{\omega}$ as a closed normal subgroup of the free real group $\hat{D}_{e, f}$, where $e \geq 1$ and $f \geq 2$.

Now it is well known that $K$ has an algebraic PRC extension $K_{\sigma}$ such that $G\left(K_{\sigma}\right) \cong \hat{D}_{e, f}$ [5]. By what has been said above $K_{\sigma}$ has an algebraic extension $E$ such that $G(E) \cong G$. By the Prestel extension theorem $E$ is also PRC.

1. Boolean spaces of countable weight. A topological space $X$ is a Boolean space if it is an inverse limit of finite discrete spaces. Two other equivalent definitions are:

(a) $X$ is a compact totally disconnected Hausdorff space;

(b) $X$ is compact and every $x \in X$ has a basis of closed-open neighbourhoods, whose intersection is $\{x\}$.

The following lemma characterizes a subclass of Boolean spaces. Here an inverse limit of topological spaces $X=\lim X_{i}$, where $i$ ranges over $\mathbf{N}=\{1,2, \ldots\}$ with its usual order, is said to be the inverse limit of a sequence of spaces. We leave the proof of the lemma to the reader. Our suggestion is to prove the implications (a) $\Rightarrow(b) \Rightarrow(c) \Rightarrow(d) \Rightarrow$ (a) and (c) $\Rightarrow(\mathrm{e}) \Rightarrow(\mathrm{f}) \Rightarrow(\mathrm{b})$. 
LEMMA 1.1. The following conditions on a Boolean space $X$ are equivalent:

(a) X has a countable basis for its topology;

(b) the family of closed-open subsets of $X$ is countable;

(c) $X$ is the inverse limit of a sequence of finite discrete spaces;

(d) $X$ is homeomorphic to a closed subset of the product space $\{ \pm 1\}^{\mathbf{N}}$;

(e) given two continuous surjections $\varphi:\{ \pm 1\}^{\mathbf{N}} \rightarrow X_{0}$ and $\alpha: X \rightarrow X_{0}$ onto a finite discrete space $X_{0}$, there exists a continuous surjection $\gamma$ : $\{ \pm 1\}^{\mathbf{N}} \rightarrow X$ such that $\alpha \circ \gamma=\varphi$

(f) either $X=\varnothing$ or $X$ is a quotient space of $\{ \pm 1\}^{\mathbf{N}}$, i.e., there exists a continuous surjection $\{ \pm 1\}^{\mathbf{N}} \rightarrow X$.

A Boolean space satisfying either of the conditions of Lemma 1.1 is said to be of weight $\leq \boldsymbol{\aleph}_{0}[\mathbf{6}, \S 2]$.

We now focus our attention on one of these spaces. The equivalence of (a) and (b) in the following lemma follows from [7, Corollary 2-98 and Corollary 2-59], that of (b) and (c) from [4, IV.4.1]. We leave the implications (a) $\Leftrightarrow$ (d). (c) \& (d) $\Rightarrow$ (e), (e) $\Rightarrow$ (a), (c) $\Rightarrow$ (f) and (f) $\Rightarrow$ (a) to the reader.

LEMMA 1.2. The following conditions on a nonempty Boolean space $X_{\omega}$ of weight $\leq \boldsymbol{\aleph}_{0}$ are equivalent.

(a) $X_{\omega}$ is perfect, i.e., has no isolated points.

(b) $X_{\omega}$ is homeomorphic to the Cantor 'middle thirds' set.

(c) $X_{\omega}$ is homeomorphic to $\{ \pm 1\}^{\mathbf{N}}$.

(d) $\left|X_{\omega}\right|>1$ and every nonempty closed-open subset of $X_{\omega}$ is homeomorphic to $X_{\omega}$.

(e) Let $X$ be a Boolean space of weight $\leq \boldsymbol{\aleph}_{0}$, let $X_{0}$ be a finite discrete space and let $\varphi: X_{\omega} \rightarrow X_{0}$ and $\alpha: X \rightarrow X_{0}$ be continuous maps. If $\alpha(X) \subseteq \varphi\left(X_{\omega}\right)$, then there exists a continuous injection $\gamma: X \rightarrow X_{\omega}$ such that $\varphi \circ \gamma=\alpha$.

(f) Let $\varphi: X_{\omega} \rightarrow X_{0}$ and $\alpha: X \rightarrow X_{0}$ be as in (e). If $\alpha(X)=\varphi\left(X_{\omega}\right)$, then there exists a continuous surjection $\gamma: X_{\omega} \rightarrow X$ such that $\alpha \circ \gamma=\varnothing$.

Definition 1.3. The Boolean space $X_{\omega}$ satisfying one, and hence all, of the conditions of Lemma 1.2 is called the universal Boolean space of weight $\boldsymbol{\aleph}_{0}$.

2. The group $\hat{D}_{\omega}$. Real free groups have been introduced in [6]. In this work we are interested in real free groups of countable rank. Among them there is a universal one denoted $\hat{D}_{\omega}$. In the notation of $[6, \S 1]$ it is 
defined as

$$
\hat{D}_{\omega}=\hat{D}\left(X_{\omega}, S_{\omega}\right)=\hat{D}\left(X_{\omega}, X_{\omega}, 1\right),
$$

where $X_{\omega}$ is the universal Boolean space of weight $\boldsymbol{\aleph}_{0}$ and $Y_{\omega}$ is the one point compactification of a countable discrete space $S_{\omega}$ (and 1 is the compactification point of $Y_{\omega}$ ). In other words $\hat{D}_{\omega}$ contains the spaces $X_{\omega}$ and $Y_{\omega}$ as disjoint closed subspaces, the elements of $X_{\omega}$ are involutions of $\hat{D}_{\omega}$ (i.e. of order 2) and the following universal property is satisfied.

(1) Every continuous map $\varphi$ from $X_{\omega} \cup Y_{\omega}$ into a profinite group $G$, such that $\varphi(x)^{2}=1$ for every $x \in X_{\omega}$ and $\varphi(1)=1$; uniquely extends to a homomorphism $\varphi: \hat{D}_{\omega} \rightarrow G$.

The following properties of $\hat{D}_{\omega}$ follow from the study of real free groups in [6].

(2) $\operatorname{rank}\left(\hat{D}_{\omega}\right)=\boldsymbol{\aleph}_{0}$ (since both $X_{\omega}$ and $Y_{\omega}$ are of weight $\boldsymbol{\aleph}_{0}$, by $[\mathbf{6}$, Lemma 2.2]).

(3) Inv $\hat{D}_{\omega}$ is closed in $\hat{D}_{\omega}$, and $X_{\omega}$ is a closed system of representatives of the distinct conjugacy classes of Inv $\hat{D}_{\omega}$ [6, Corollaries 3.2 and 3.3].

(4) $\hat{D}_{\omega}$ is real projective, i.e., if $\alpha: B \rightarrow A$ is an epimorphism of finite groups and $\varphi: \hat{D}_{\omega} \rightarrow A$ is a homomorphism and if for every $\varepsilon \in \operatorname{Inv} \hat{D}_{\omega}$ such that $\varphi(\varepsilon) \neq 1$ there exists a $b \in \operatorname{Inv} B$ such that $\alpha(b)=\varphi(\varepsilon)$, then there exists a homomorphism $\gamma: \hat{D}_{\omega} \rightarrow B$ such that $\alpha \circ \gamma=\varphi[\mathbf{6}$, Corollary 3.3].

(5) The subsets $Y_{\omega}$ and $S_{\omega}$ of $\hat{D}_{\omega}$ converge to 1 , i.e., for every open subgroup $N$ of $\hat{D}_{\omega}$ the set $Y_{\omega}-N=S_{\omega}-N$ is finite (since it is closed in $Y_{\omega}$, hence compact, and discrete).

(6) Every closed subgroup of $\hat{D}_{\omega}$ is a real projective group of rank $\leq \boldsymbol{\aleph}_{0}$ (by (2) and [5, Corollary 10.5]).

(7) the following characterization of $\hat{D}_{\omega}$ makes it especially attractive:

Proposition 2.1. Let $R$ be a countable real closed field and let $F=R(t)$ be the field of rational functions in one variable over $R$. Then $G(F) \cong \hat{D}_{\omega}$.

Proof. By [6, Proposition 4.1], $G(F) \cong \hat{D}(X(F), H)$ where $X(F)$ is the space of orderings of $F$ and $H=\{a+b \sqrt{-1} \mid a, b \in R$ and $b>0\}$. By [2, Theorem 11], $X(F) \cong X_{\omega}$; also $|H|=\boldsymbol{\aleph}_{0}=\left|S_{\omega}\right|$. Hence $\hat{D}(X(F), H) \cong \hat{D}\left(X_{\omega}, S_{\omega}\right)=\hat{D}_{\omega}$.

We shall see that the converse of the property (6) above is also true. But first we need a lemma, which is an easy corollary of some of the deeper theorems of [5]. It appears as Lemma 3.5 of [6]. 
LEMMA 2.2. Let $P$ and $G$ be real projective groups.

(a) There exists a closed system of representatives of the conjugacy classes of $\operatorname{Inv}(G)$.

(b) Let $\alpha: P \rightarrow G$ be a continuous epimorphism and let $X$ be a system of representatives of the conjugacy classes of $\operatorname{Inv}(P)$. If $\alpha$ maps $X$ bijectively onto a system of representatives of the conjugacy classes of $\operatorname{Inv}(G)$, then there exists a continuous monomorphism $\gamma: G \rightarrow P$ such that $\alpha \circ \gamma=\mathrm{id}_{G}$.

Proposition 2.3. Let $\varphi: \hat{D}_{\omega} \rightarrow H$ be an epimorphism onto a finite group $H$ and let $G$ be a real projective group of rank $\leq \aleph_{0}$. If $\pi: G \rightarrow H$ is an epimorphism such that $\pi(\operatorname{Inv} G) \subseteq \varphi\left(\operatorname{Inv} \hat{D}_{\omega}\right)$ then there exists an embedding $\gamma: G \rightarrow \hat{D}_{\omega}$ such that $\varphi \circ \gamma=\pi$.

Proof. Note that the existence of a homomorphism $\gamma: G \rightarrow \hat{D}_{\omega}$ such that $\varphi \circ \gamma=\pi$ is already guaranteed by the definition of real projective groups. Our task is to use the assumption on the weight of $G$ and to show how to choose $\gamma$ to be injective. Our proof breaks into parts.

Part A: Choosing a more convenient basis for $\hat{D}_{\omega}$.

By Lemma 2.2(a), there exists a closed system $X$ of representatives of the conjugacy classes of $\operatorname{Inv} G$.

Claim. With no loss we may assume that $\pi(X) \subseteq \varphi\left(X_{\omega}\right)$ and $H=$ $\varphi\left\langle Y_{\omega}\right\rangle$.

Indeed, for each $h \in \pi(X)$ we may choose a $g=g(h) \in G$ such that $h^{\pi(g)} \in \varphi\left(X_{\omega}\right)$, since $\pi(X) \subseteq \pi(\operatorname{Inv} G) \subseteq \varphi\left(\operatorname{Inv} \hat{D}_{\omega}\right)$ and $X_{\omega}$ is a system of representatives of the conjugacy classes of $\hat{D}_{\omega}$, by (3). We replace then $X$ by

$$
\tilde{X}=\bigcup_{h \in \pi(X)}\left\{x^{g(h)} \mid x \in X, \pi(x)=h\right\},
$$

which is also a closed system of representatives of the conjugacy classes of Inv $G$ and satisfies

$$
\pi(\tilde{X})=\left\{h^{\pi(g(h))} \mid h \in \pi(X)\right\} \subseteq \varphi\left(X_{\omega}\right) .
$$

Let $x_{1}, \ldots, x_{n} \in X_{\omega}$ such that $\varphi\left(X_{\omega}\right)=\left\{\varphi\left(x_{1}\right), \ldots, \varphi\left(x_{n}\right)\right\}$. By (5), $Y_{\omega} \cap \operatorname{Ker}(\varphi)$ is infinite, hence we can choose $n$ elements $y_{1}, \ldots, y_{n} \in\left(Y_{\omega}\right.$ $\cap \operatorname{Ker} \varphi)-\{1\}$. Let $Y_{0}=Y_{\omega}-\left\{y_{1}, \ldots, y_{n}\right\}$ and $Y_{1}=\left\{x_{1} y_{1}, \ldots, x_{n} y_{n}\right\}$. Then

$$
\begin{aligned}
H & =\left\langle\varphi\left(X_{\omega}\right), \varphi\left(Y_{\omega}\right)\right\rangle=\left\langle\varphi\left(x_{1}\right), \ldots, \varphi\left(x_{n}\right), \varphi\left(Y_{0}\right)\right\rangle \\
& =\left\langle\varphi\left(x_{1} y_{1}\right), \ldots, \varphi\left(x_{n} y_{n}\right), \varphi\left(Y_{0}\right)\right\rangle=\varphi\left\langle Y_{0} \cup Y_{1}\right\rangle .
\end{aligned}
$$


Define $\rho: X_{\omega} \cup Y_{\omega} \rightarrow \hat{D}_{\omega}$ by

$$
\begin{array}{ll}
\rho(x)=x & \text { for } x \in X_{\omega} \\
\rho(y)=y & \text { for } y \in Y_{0}, \text { and } \\
\rho\left(y_{i}\right)=x_{\imath} y_{\imath} & \text { for } i=1, \ldots, n .
\end{array}
$$

Then $\rho$ extends to a homomorphism $\rho: \hat{D}_{\omega} \rightarrow \hat{D}_{\omega}$. But $\rho^{2}(z)=z$ for all $z \in X_{\omega} \cup Y_{\omega}$, hence $\rho^{2}$, and therefore also $\rho$, is an isomorphism. It follows that $\left(X_{\omega}, Y_{0} \cup Y_{1}\right)$ is also a basis for $\hat{D}_{\omega}$. Thus we may replace $Y_{\omega}$ by $Y_{0} \cup Y_{1}$ and attain the required property. This proves the Claim.

\section{Part B: Constructing $\gamma$.}

By Lemma 1.2(e) there exists a continuous embedding $\beta: X \rightarrow X_{\omega}$ such that $\varphi \circ \beta(x)=\pi(x)$ for each $x \in X$. Let $X^{\prime}=\beta(X)$ and let $\alpha$ : $X^{\prime} \rightarrow X$ be the inverse of $\beta$; then $\pi \circ \alpha(x)=\varphi\left(x^{\prime}\right)$ for each $x^{\prime} \in X^{\prime}$.

By [6, Proposition 3.4] (or by a direct check), the group $\left\langle Y_{\omega}\right\rangle$ is real free with the basis $\left(\varphi, Y_{\omega}\right)$, i.e., $\left\langle Y_{\omega}\right\rangle \cong \hat{F}_{\omega}$ is the free profinite group of rank $\aleph_{0}$. Since $\varphi\left\langle Y_{\omega}\right\rangle=H$, by the Iwasawa theorem ([12, p. 84]), there exists a continuous epimorphism $\hat{\alpha}:\left\langle Y_{\omega}\right\rangle \rightarrow G$ such that $\pi \circ \hat{\alpha}(y)=\varphi(y)$ for every $y \in\left\langle Y_{\omega}\right\rangle$. Denote by $\alpha$ its restriction to $Y_{\omega}$.

Let $Z=X^{\prime} \cup Y_{\omega}$. We have constructed a continuous map $\alpha: Z \rightarrow G$ such that $\pi \circ \alpha=\varphi$ on $Z$. Let $\hat{D}=\langle Z\rangle$. By [6, Proposition 3.4], $\hat{D}$ is a real free group and $\left(X^{\prime}, Y_{\omega}\right)$ is its basis, hence $\alpha$ extends to a homomorphism $\alpha: \hat{D} \rightarrow G$ such that $\pi \circ \alpha=\varphi$, on $\hat{D}$. Clearly the restriction of $\alpha$ to $\left\langle Y_{\omega}\right\rangle$ is $\hat{\alpha}$, hence $\alpha$ is an epimorphism. By [6, corollary 3.2], $X^{\prime}$ is a closed system of representatives of the conjugacy classes of $\operatorname{Inv} \hat{D}$ and it maps bijectively onto $X$ by $\alpha$. Therefore by Lemma 2.2(b), there exists an embedding $\gamma: G \rightarrow \hat{D}$ such that $\alpha \circ \gamma=\mathrm{id}_{G}$. Clearly $\pi=\varphi \circ \gamma$.

3. Characterization of $\hat{D}_{\omega}$ by embedding problems. A proper real embedding problem for a profinite group $G$ is a diagram

$$
\begin{aligned}
& G \\
& \downarrow \rightarrow \varphi \\
\rightarrow & A
\end{aligned}
$$

and a closed involution domain $I$ (i.e., a closed subset $I \subseteq \operatorname{Inv} B$ closed under conjugation) of $B$ such that $\pi$ and $\varphi$ are epimorphisms of profinite groups and $\pi(I)=\varphi(\operatorname{Inv} G)$.

A solution to the problem is an epimorphism $\gamma: G \rightarrow B$ such that $\pi \circ \gamma=\varphi$ and $\gamma(\operatorname{Inv} G)=I$.

Problem (1) is said to be finite if $B$ is a finite group. 
REMARK 3.1. If every finite proper real embedding problem for a profinite group $G$ is solvable, then $\operatorname{Inv} G \neq \varnothing$ and $G$ has an open subgroup $G^{\prime}$ of index 2 such that $G^{\prime} \cap \operatorname{Inv}(G)=\varnothing$. In particular $\operatorname{Inv} G$ is closed in $G$.

Indeed, consider the diagram

$$
\begin{array}{r}
G \\
\downarrow \\
\{ \pm 1\} \rightarrow\{1\}
\end{array}
$$

and the involution domain $\{-1\}$ of $\{ \pm 1\}$. By assumption there exists an epimorphism $\gamma: G \rightarrow\{ \pm 1\}$ such that $\gamma(\operatorname{Inv} G)=\{-1\}$. Then $G^{\prime}=\operatorname{Ker} \gamma$ has the required properties.

LEMMA 3.2. Every finite proper real embedding problem for $\hat{D}_{\omega}$ is solvable.

Proof. Let diagram (1) with an involution domain $I \subseteq B$ be a finite problem for $G=\hat{D}_{\omega}$. Denote $I_{0}=\left\{b \in I \mid \pi(b) \in \varphi\left(X_{\omega}\right)\right\}$; then every element of $I$ is conjugate to an element of $I_{0}$, since every element of Inv $\hat{D}_{\omega}$ is conjugate to an element of $X_{\omega}$ and $\pi(I)=\varphi\left(\operatorname{Inv} \hat{D}_{\omega}\right)$. The set $Y_{1}=Y_{\omega}-\operatorname{Ker}(\varphi)$ is finite. Choose a subset $Y_{2} \subseteq Y_{\omega}-\left(Y_{1} \cup\{1\}\right)$ of exactly $|\operatorname{Ker} \pi|$ elements and denote $Y_{3}=Y_{\omega}-\left(Y_{1} \cup Y_{2}\right)$.

By Lemma 1.2(f), there exists a continuous surjection $\gamma_{0}: X_{\omega} \rightarrow I_{0}$ such that $\pi \circ \gamma_{0}=\varphi$ on $X_{\omega}$. Define $\gamma_{1}: Y_{1} \rightarrow B$ such that $\pi \circ \gamma_{1}=\varphi$ on $Y_{1}$ and let $\gamma_{2}: Y_{2} \rightarrow \operatorname{Ker} \pi$ be a bijection. Finally define $\gamma_{3}: Y_{3} \rightarrow B$ by $\gamma_{3}\left(Y_{3}\right)=1$. The map $\gamma: X_{\omega} \cup Y_{\omega} \rightarrow B$ that extends these four maps is continous and $\pi \circ \gamma=\varphi$ on $X_{\omega} \cup Y_{\omega}$. Therefore the unique homomorphism $\gamma: \hat{D}_{\omega} \rightarrow B$ that extends it satisfies $\pi \circ \gamma=\varphi$. Moreover, Ker $\pi \subseteq$ $\gamma\left(\hat{D}_{\omega}\right)$ and $\varphi\left(\hat{D}_{\omega}\right)=\pi(B)=A$, hence $\gamma$ is an epimorphism. This also implies that $\gamma(\operatorname{Inv} \hat{D})$ is the smallest involution domain that contains $\gamma\left(X_{\omega}\right)=I_{0}$, hence $\gamma(\operatorname{Inv} \hat{D})=I$.

We strengthen Lemma 3.2 by going to a countable inverse limit.

COROLlaRY 3.3. Consider the following diagram

$$
\underset{\pi}{B \underset{\pi}{ } \stackrel{\hat{D}_{\omega}}{ }{ }^{\downarrow} \boldsymbol{A}}
$$


in which $A$ is a finite group, $B$ is a profinite group of rank $\leq \boldsymbol{\aleph}_{0}$, and $\varphi$ and $\pi$ are epimorphisms. Let $I$ be a closed involution domain in $B$ such that $\pi(I)=\varphi\left(\operatorname{Inv} \hat{D}_{\omega}\right)$. Then there exists an epimorphism $\gamma: \hat{D}_{\omega} \rightarrow B$ such that $\pi \circ \gamma=\varphi$ and $\gamma\left(\operatorname{Inv} \hat{D}_{\omega}\right)=I$.

Proof. Let Ker $\pi \geq N_{1} \geq \mathrm{N}_{2} \geq \mathrm{N}_{3} \geq \cdots$ be a descending sequence of open normal subgroups with a trivial intersection. Let $\pi_{n}: B / N_{n} \rightarrow A$ be the epimorphism induced by $\pi$, then $I_{n}=I / N_{n}$ is an involution domain of $B / N_{n}$ and $\pi_{n}\left(I_{n}\right)=\varphi\left(\operatorname{Inv} \hat{D}_{\omega}\right)$. Assume by induction, that there exists an epimorphism $\gamma_{n}: \hat{D}_{\omega} \rightarrow B / N_{n}$ such that $\pi_{n} \circ \gamma_{n}=\varphi$ and $\gamma_{n}\left(\operatorname{Inv} \hat{D}_{\omega}\right)=$ $I_{n}$. Let $\beta_{n}: B / N_{n+1} \rightarrow B / N_{n}$ be the canonical epimorphism. Then, by Lemma 3.2, there exists an epimorphism $\gamma_{n+1}: \hat{D}_{\omega} \rightarrow B / N_{n+1}$ such that $\beta_{n} \circ \gamma_{n+1}=\gamma_{n}$ and $\gamma_{n+1}\left(\operatorname{Inv} \hat{D}_{\omega}\right)=I_{n+1}$, hence $\pi_{n+1} \circ \gamma_{n+1}=\varphi$.

The epimorphisms $\gamma_{n}$ define an epimorphism $\gamma: \hat{D}_{\omega} \rightarrow B$ such that $\pi \circ \gamma=\varphi$ and $\varphi\left(\operatorname{Inv} \hat{D}_{\omega}\right)=I$, since $I=\lim _{\leftarrow} I_{n}$.

The converse of Lemma 3.2 is also true:

LEMMA 3.4. Let $G$ be a profinite group of rank $\leq \boldsymbol{\aleph}_{0}$. If every finite proper real embedding problem for $G$ is solvable then $G \cong \hat{D}_{\omega}$.

Proof. Both $G$ and $\hat{D}_{\omega}$ have descending sequences of open normal subgroups whose intersections are 1 , say $G=N_{1}^{\prime} \geq N_{2}^{\prime} \geq \cdots$ and $\hat{D}_{\omega}=$ $M_{1}^{\prime} \geq M_{2}^{\prime} \geq \cdots$. Let $n \geq 0$ and assume by induction that we have already constructed

(a) two sequences of open normal subgroups $G=N_{0} \geq N_{1} \geq N_{2} \geq$ $\cdots \geq N_{n}$ and $\hat{D}_{\omega}=M_{0} \geq M_{1} \geq M_{2} \geq \cdots \geq M_{n}$ such that $N_{i} \leq N_{l}^{\prime}$ and $M_{l} \leq M_{i}^{\prime}$ for $i=1, \ldots, n$, and

(b) isomorphisms $\varphi_{i}: G / N_{\imath} \rightarrow \hat{D}_{\omega} / M_{i}$ such that $\varphi_{l}\left((\operatorname{Inv} G) / N_{\imath}\right)=$ $\left(\operatorname{Inv} \hat{D}_{\omega}\right) / M_{i}$ for $i=0,1, \ldots, n$ and the following diagrams commute

$$
G / N_{l} \quad \stackrel{\varphi_{t}}{\rightarrow} \quad \hat{D}_{\omega} / M_{i}
$$

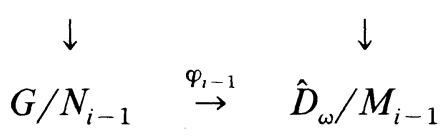

for $i=1, \ldots, n$ (where the vertical maps are the canonical epimorphisms). Note that the trivial map $\varphi_{0}: G / N_{0} \rightarrow \hat{D}_{\omega} / M_{0}$ satisfies (b), since $\operatorname{Inv} G$ and Inv $\hat{D}_{\omega}$ are not empty, by Remark 3.1. 
Let $K=N_{n+1}^{\prime} \cap N_{n}$. By Lemma 3.2 there exists an epimorphism $\gamma^{\prime}$ : $\hat{D}_{\omega} \rightarrow G / K$ such that

$$
\begin{array}{ccc} 
& \hat{D}_{\omega} \\
\gamma^{\prime} \swarrow & \downarrow \\
G / K \rightarrow G / N_{n} \stackrel{\varphi_{n}}{\rightarrow} \hat{D}_{\omega} / M_{n}
\end{array}
$$

commutes and $\gamma^{\prime}\left(\operatorname{Inv} \hat{D}_{\omega}\right)=(\operatorname{Inv} G) / K$. Let $M_{n+1}=N_{n+1}^{\prime} \cap\left(\operatorname{Ker} \gamma^{\prime}\right)$ and let $\gamma: \hat{D}_{\omega} / M_{n+1} \rightarrow G / K$ be the epimorphism induced by $\gamma^{\prime}$. Then $M_{n+1} \leq M_{n} \cap M_{n+1}^{\prime}$,

$$
\begin{array}{ccc}
G / K & \stackrel{\gamma}{\leftarrow} & \hat{D}_{\omega} / M_{n+1} \\
\downarrow & & \downarrow \\
G / N_{n} & \stackrel{\varphi_{n}}{\rightarrow} & \hat{D}_{\omega} / M_{n}
\end{array}
$$

commutes and $\gamma\left(\left(\operatorname{Inv} \hat{D}_{\omega}\right) / M_{n+1}\right)=(\operatorname{Inv} G) / K$. By assumption there exists an epimorphism $\varphi^{\prime}: \quad G \rightarrow \hat{D}_{\omega} / M_{n+1}$ such that $\varphi^{\prime}(\operatorname{Inv} G)=$ $\left(\operatorname{Inv} \hat{D}_{\omega}\right) / M_{n+1}$ and

$$
\begin{aligned}
& G \\
& \downarrow \quad \searrow \varphi^{\prime} \\
& G / K \stackrel{\gamma}{\leftarrow} \hat{D}_{\omega} / M_{n+1}
\end{aligned}
$$

commutes. Let $N_{n+1}=\operatorname{Ker} \varphi^{\prime}$ and let $\varphi_{n+1}: G / N_{n+1} \rightarrow \hat{D} / M_{n+1}$ be the isomorphism induced by $\varphi^{\prime}$. Then $N_{n+1} \leq N_{n+1}^{\prime} \cap N_{n}$, diagram (2) commutes for $i=n+1$ and $\varphi_{n+1}\left((\operatorname{Inv} G) / N_{n+1}\right)=\left(\operatorname{Inv} \hat{D}_{\omega}\right) / M_{n+1}$.

The compatible sequence $\varphi_{0}, \varphi_{1}, \varphi_{2}, \ldots$ of isomorphisms defines an isomorphism $\varphi: G \rightarrow \hat{D}_{\omega}$.

4. Embedding of $\hat{D}_{\omega}$ in $\hat{D}_{e, f}$. The aim of this section is to identify $\hat{D}_{\omega}$ as a closed normal subgroup of a finitely generated real free group $\hat{D}_{e, f}$, where $e, f \geq 0$. Recall $[6, \S 1]$ that $\hat{D}_{e, f}=\hat{D}(X, S)$, where $X$ and $S$ are the discrete spaces of $e$ and $f$ elements, respectively. In other words, there are $\varepsilon_{1}, \ldots, \varepsilon_{e}, \sigma_{1}, \ldots, \sigma_{f} \in \hat{D}_{e, f}$ such that

(1) $\hat{D}_{e, f}=\left\langle\varepsilon_{1}, \ldots, \varepsilon_{e}, \sigma_{1}, \ldots, \sigma_{f}\right\rangle, \varepsilon_{1}^{2}=\cdots=\varepsilon_{e}^{2}=1$; and

(2) every map $\vartheta$ from $\left\{\varepsilon_{1}, \ldots, \varepsilon_{e}, \sigma_{1}, \ldots, \sigma_{f}\right\}$ into a profinite group $G$ such that $\vartheta\left(\varepsilon_{1}\right)^{2}=\cdots=\vartheta\left(\varepsilon_{e}\right)^{2}=1$, uniquely extends to a homomorphism $\vartheta: \hat{D}_{e, f} \rightarrow G$. 
LEMMA 4.1. Let $\varepsilon_{1}, \ldots, \varepsilon_{e}, \sigma_{1}, \ldots, \sigma_{f} \in \hat{D}_{e, f}$ satisfy (1). Then:

(a) the property (2) is also satisfied.

(b) for every $\varepsilon \in \operatorname{Inv} \hat{D}_{e, f}$ there exists a unique $1 \leq i \leq e$ such that $\varepsilon$ is conjugate to $\varepsilon_{i}$; moreover, $\left\{\sigma \in \hat{D}_{e, f} \mid \varepsilon^{\sigma}=\varepsilon\right\}=\{1, \varepsilon\}$.

(c) $\hat{D}_{e, f}=\hat{D}_{e} * \hat{F}_{f}$, where $\hat{D}_{e}=\left\langle\varepsilon_{1}, \ldots, \varepsilon_{e}\right\rangle$ is the free (profinite) product of $e$ copies of $\mathbf{Z} / 2 \mathbf{Z}$ and $\hat{F}_{f}=\left\langle\sigma_{1}, \ldots, \sigma_{f}\right\rangle$ is the free profinite group of $\operatorname{rank} f$.

Proof. (a) see [5, the remark preceding Lemma 5.4].

(b) see [5, Proposition 6.1].

(c) follows from (2).

The following assertion is used in the sequel. Its proof is left to the reader.

LEMMA 4.2. Let $\varphi: G \rightarrow H$ be an epimorphism of profinite groups and let $S$ be a subset of $G$. If $G_{0}$ is the smallest closed normal subgroup of $G$ containing $S$, then $\varphi\left(G_{0}\right)$ is the smallest closed normal subgroup of $H$ containing $\varphi(S)$.

The key to the Lubotzky-v.d. Dries method [10] of recognizing certain closed subgroups of $\hat{F}_{m}$ as isomorphic to $\hat{F}_{\omega}$ is the Nielsen-Schreier formula for the rank of open subgroups of $\hat{F}_{m}$. The same role is played in our context by the following special case of the Binz-Neukirch-Wenzel theorem [1, p. 105].

LEMMA 4.3. Let $G=\Re_{\imath \in I} G_{l}$ be the free product of the profinite groups $G_{i}$, where $I$ is a finite set. Let $H$ be an open subgroup of $G$. For every $i \in I$ we consider the double class decomposition of $G$ :

$$
G=\bigcup_{J \in J(i)} G_{i} x(i, j) H .
$$

Then

$$
H \cong \prod_{i} \prod_{j \in J(i)}\left(G_{l}^{x(l, J)} \cap H\right) * \hat{F}_{m},
$$

where

$$
m=\sum_{i \in I}[(G: H)-|J(i)|]-(G: H)+1 .
$$

Corollary 4.4. Suppose that $G=D * F$, where $D \cong \hat{D}_{e}$ and $F \cong \hat{F}_{f}$. If $H$ is an open normal subgroup of $G$ of index $n$ which contains $D$, then $H \cong \hat{D}_{e n, 1+n(f-1)}$. 
Proof. For $z \in G$ we have $D z H=D H z=H z$, hence if $G=$ $\bigcup_{i=1}^{n} H z(i)$, then $G=\bigcup_{i=1}^{n} D z(i) H$. Moreover, $D^{z(i)} \cap H=D^{z(i)} \cong \hat{D}_{e}$, since $H \triangleleft G$. Secondly, $F z H=F H z=G$ and $(F: F \cap H)=(G: H)=n$, hence, by the Nielsen-Schreier formula, [1, p. 108] $F \cap H \cong \hat{F}_{1+n(f-1)}$. Thus, in the notation of Lemma 4.2

and

$$
m=[(G: H)-n]+[(G: H)-1]-(G: H)+1=0
$$

$$
\begin{aligned}
H & \cong\left(D^{z(1)} \cap H\right) *\left(D^{z(2)} \cap H\right) * \cdots *\left(D^{z(n)} \cap H\right) *(F \cap H) \\
& =D^{z(1)} * D^{z(2)} * \cdots * D^{z(n)} *(F \cap H) \cong \hat{D}_{e n} * \hat{F}_{1+n(f-1)} .
\end{aligned}
$$

Proposition 4.5. Let $G=\hat{D}_{e, f}$, where $e \geq 1$ and $f \geq 2$, and let $K$ be an open subgroup of $G$. Then there exists a closed normal subgroup $H$ of $G$ such that $H \cong \hat{D}_{\omega}, G=K H$ and

$$
\operatorname{Inv} H=\operatorname{Inv} G .
$$

Proof. We break the proof into parts.

Part A. Construction of $H$.

By Lemma 4.1(c), $G=D * F$, where $D \cong \hat{D}_{e}$ and $F \cong \hat{F}_{f}$ are closed subgroups of $G$. Let $p$ be a prime which does not divide $(G: K)$. Fix an epimorphism $\rho: G \rightarrow \mathbf{Z}_{p}$ such that $\rho(D)=1$ and let $H=\operatorname{Ker} \rho$. The lattice $G=G_{0}>G_{1}>G_{2}>\cdots$ of open subgroups of $G$ containing $H$ is isomorphic to the lattice of open subgroups of $\mathbf{Z}_{p}$, hence $G_{i}$ is the only normal subgroup of $G$ of index $p^{i}$ containing $H$, for each $i \geq 0$, and $H=\bigcap_{i=0}^{\infty} G_{i}$. In particular $(G: K H)=p^{i}$ for some $i \geq 0$, but $(G: K H) \mid(G: K)$, hence $i=0$, whence $G=K H$. By Lemma 4.1(b), every involution of $G=D * F$ is conjugate to an involution of $D$; but $D \leq H$, hence (3) holds.

Part B. Embedding problem.

We employ Lemma 3.4 to show that $H \cong \hat{D}_{\omega}$. Let

$$
\begin{aligned}
& \text { H } \\
& \underset{\pi}{\rightarrow} A
\end{aligned}
$$

together with an involution domain $I \subseteq \operatorname{Inv} B$ such that $\pi(I)=\varphi(\operatorname{Inv} H)$ be a finite proper embedding problem for $H$. Then $\operatorname{Ker} \varphi$ is open in $H$, hence there exists an open $N \triangleleft G$ such that $N \cap H=\operatorname{Ker} \varphi$. Now $H \leq$ $N H \triangleleft G$, hence there is an $i \geq 0$ such that $N H=G_{i}$. It follows that $\varphi$ can be extended to an epimorphism $\varphi: G_{\imath} \rightarrow A$, with kernel $N$. 
Let $n=p|B|+i$. With no loss we may assume that $i=0$, i.e., $\varphi$ can be extended to $G$, and, moreover, that

$$
f>2 n
$$

and

(6) for every conjugacy class $C \subseteq \varphi(\operatorname{Inv} H)$ there are at least $n$ conjugacy classes in $\operatorname{Inv} G$ mapped by $\varphi$ onto $C$ (in particular $e \geq n$, by Lemma $4.1(\mathrm{~b}))$.

Indeed, otherwise replace $G$ by its open normal subgroup $G_{n}$ (and $G_{j}$ by $G_{j+n}$ for every $j \geq 0$ and $N$ by $\left.G_{n} \cap N\right)$ and restrict $\varphi$ from $G_{l}$ to $G_{n}$. By Corollary $4.4, G_{n} \cong \hat{D}_{e^{\prime}, f^{\prime}}$, where

$$
f^{\prime}=1+p^{n}(f-1)>2^{n} \geq 2 n \text {. }
$$

Also, $\rho\left(G_{n}\right) \cong \mathbf{Z}_{p}$ and Inv $G_{n} \subseteq H$, by (3). To check (6) we consider an $\varepsilon \in \operatorname{Inv} H$ which satisfies $\varphi(\varepsilon) \in C$, and let $\sigma(1), \ldots, \sigma(n) \in G$ belong to distinct cosets modulo $G_{n}$. Then $\varepsilon^{\sigma(1)}, \ldots, \varepsilon^{\sigma(n)} \in \varphi^{-1}(C)$ represent distinct conjugacy classes in $G_{n}$. Indeed, if $\varepsilon^{\sigma(j)}=\varepsilon^{\sigma(k) \tau}$, where $1 \leq j$, $k \leq n$ and $\tau \in G_{n}$, then $\sigma(k) \tau \sigma(j)^{-1}=1$ or $\sigma(k) \tau \sigma(j)^{-1}=\varepsilon$, by Lemma 4.1(b). In both cases $\sigma(k) \tau \sigma(j)^{-1} \in G_{n}$, whence $\sigma(k) G_{n}=\sigma(j) G_{n}$, i.e., $k=j$.

In particular, $G=N H$, which implies that

$$
G=N G_{1} \text {. }
$$

Part C. Generators for $G$.

Let $H_{0}, A_{0}$ and $B_{0}$ be the smallest closed normal subgroups of $G, A$ and $B$ containing $\operatorname{Inv} G=\operatorname{Inv} H, \varphi(\operatorname{Inv} H)=\pi(I)$ and $I$, respectively. By Lemma 4.3 we have

$$
\varphi\left(H_{0}\right)=A_{0}=\pi\left(B_{0}\right) .
$$

Also $D \leq H_{0} \leq H$, since $D$ is generated by involutions. Let us show that

$$
\left(F \cap H_{0} N\right)-G_{1} \neq \varnothing \text {. }
$$

Indeed, $G_{1} \neq G$, hence $N \nless G_{1}$, by (7). Also, $G=H_{0} F$, since $G=$ $\langle D, F\rangle$, by Lemma 4.1(c) and $D \leq H_{0}$. Thus there exists a $\sigma \in N-G_{1}$, and there exists an $\varepsilon \in H_{0}$ such that $\varepsilon \sigma \in F$. Clearly $\varepsilon \sigma \notin G_{1}$, hence $\varepsilon \sigma \in\left(F \cap H_{0} N\right)-G_{1}$.

We use (9) to find generators for $F$. Let $\sigma \mapsto \bar{\sigma}$ denote the canonical map $G \rightarrow \bar{G}$, where $\bar{G}=G / N \cap G_{1}$. By (7)

$$
\bar{G} \cong G / N \times G / G_{1} \cong A \times \mathbf{Z} / p \mathbf{Z},
$$

hence $|\bar{G}|=p|A| \leq n$. Choose generators $\bar{\sigma}_{1}, \ldots, \bar{\sigma}_{n}$ of the subgroup $\bar{F}$ of $\bar{G}$. With no loss $\bar{\sigma}_{1} \in \overline{\left(F \cap H_{0} N\right)-G_{1}}$, by (9). Put $\bar{\sigma}_{n+1}=\cdots=\bar{\sigma}_{f}=1$. By the Gaschütz Lemma [9, Lemma 4.2], $\bar{\sigma}_{1}, \ldots, \bar{\sigma}_{f}$ lift to generators 
$\sigma_{1}, \ldots, \sigma_{f}$ of $F$. Observe that

$$
\sigma_{1} \notin G_{1}
$$

and $\varphi\left(\sigma_{1}\right) \in \varphi\left(H_{0} N\right)=\varphi\left(H_{0}\right)$, hence by (8)

$$
\varphi\left(\sigma_{1}\right) \in \pi\left(B_{0}\right)
$$

also

$$
\varphi\left(\sigma_{n+1}\right)=\cdots=\varphi\left(\sigma_{f}\right)=1 .
$$

Fix $\varepsilon_{1}, \ldots, \varepsilon_{e} \in \operatorname{Inv} G$ that generate $D$. Then $G=\left\langle\varepsilon_{1}, \ldots, \varepsilon_{e}\right.$, $\left.\sigma_{1}, \ldots, \sigma_{f}\right\rangle$, by Lemma 4.1(c).

Part D. Solution by the embedding problem.

Define $\gamma:\left\{\varepsilon_{1}, \ldots, \varepsilon_{e}, \sigma_{1}, \ldots, \sigma_{f}\right\} \rightarrow B$ as follows. First choose $\gamma\left(\sigma_{1}\right), \ldots, \gamma\left(\sigma_{n}\right) \in B$ such that $\pi\left(\gamma\left(\sigma_{j}\right)\right)=\varphi\left(\sigma_{j}\right), j=1, \ldots, n$, and

$$
\gamma\left(\sigma_{1}\right) \in B_{0}
$$

(this is possible by (11)). Next let $\gamma\left(\sigma_{n+1}\right), \ldots, \gamma\left(\sigma_{f}\right)$ be a set of generators of $\operatorname{Ker} \pi$ (by (5), $|\operatorname{Ker} \pi| \leq n<n-f$ ). Finally let $b_{1}, \ldots, b_{m}$ be representatives of the conjugacy classes of $I$, with $m \leq n$. By Lemma 4.1(b) every $a \in \varphi(\operatorname{Inv} G)=\pi(I)$ is conjugate to one of the $\varphi\left(\varepsilon_{1}\right), \ldots, \varphi\left(\varepsilon_{e}\right)$; moreover, by (6), $a$ is conjugate to at least $n$ elements of this $e$-tuple. Thus, reordering $\varepsilon_{1}, \ldots, \varepsilon_{e}$ if necessary, we may assume that $\pi\left(b_{i}\right)$ is conjugate to $\varphi\left(\varepsilon_{i}\right)$, for $i=1, \ldots, m$. Therefore with no loss $\pi\left(b_{i}\right)=\varphi\left(\varepsilon_{i}\right)$, for $i=1, \ldots, m$. Choose $b_{m+1}, \ldots, b_{e} \in I$ such that $\pi\left(b_{i}\right)=\varphi\left(\varepsilon_{i}\right)$, for $i=m+1, \ldots, e$ and define $\gamma\left(\varepsilon_{i}\right)=b_{i}$, for $i=1, \ldots, e$.

The map $\gamma$ uniquely extends to a homomorphism $\gamma: G \rightarrow B$ such that $\pi \circ \gamma=\varphi$. But $\operatorname{Ker} \pi \subseteq \gamma(G)$ and $\varphi(G)=\pi(B)=A$, hence $\gamma(G)=B$. Also $\gamma\left(\varepsilon_{1}\right), \ldots, \gamma\left(\varepsilon_{0}\right) \in I$, hence $\gamma(\operatorname{Inv} G) \subseteq I$ by Lemma $4.1(\mathrm{~b})$. On the other hand $b_{1}, \ldots, b_{m} \in \gamma(\operatorname{Inv} G)$, hence $I \subseteq \gamma(\operatorname{Inv} G)$. Therefore, by (3)

$$
\gamma(\operatorname{Inv} G)=\gamma(\operatorname{Inv} H)=I \text {. }
$$

Finally, $\left\langle\sigma_{1}\right\rangle H$ is an open subgroup of $G$ containing $H$, hence $\left\langle\sigma_{1}\right\rangle H=G_{i}$ for some $i \geq 0$. But $\left\langle\sigma_{1}\right\rangle \nless G_{1}$ by (10), hence $\left\langle\sigma_{1}\right\rangle H=G_{0}$ $=G$. By Lemma 4.2 and (14), $\gamma\left(H_{0}\right)=B_{0}$, in particular $\gamma\left(\sigma_{1}\right) \in \gamma\left(H_{0}\right)$ $\subseteq \gamma(H)$, by (13). Therefore $\gamma(G)=\gamma\left(\left\langle\sigma_{1}\right\rangle H\right)=\gamma(H)$. Thus the restriction of $\gamma$ to $H$ solves the problem (4).

5. Algebraic realization of real projective groups of rank $\leq \boldsymbol{\aleph}_{0}$. We are now in a position to apply the information about real projective groups of rank $\leq \boldsymbol{\aleph}_{0}$ gathered so far to realize them as the absolute Galois groups of algebraic PRC fields. Thus is our main result. 
THEOREM 5.1. Let $K$ be a countable formally real Hilbertian field and let $K^{\prime}$ be a finite Galois extension of $K$. If $G$ is a real projective group of rank $\leq \boldsymbol{\aleph}_{0}$ and $\pi: G \rightarrow \mathscr{G}\left(K^{\prime} / K\right)$ is an epimorphism such that $\pi(\operatorname{Inv} G)$ $\subseteq \operatorname{res}_{K^{\prime}}(\operatorname{Inv} G(K))$, then there exists a PRC algebraic extension $E$ of $K$ and an isomorphism $\gamma$ such that the following diagram is commutative.
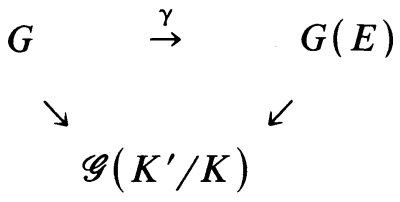

Proof. Let $\left\{\overline{\boldsymbol{\delta}}_{1}, \ldots, \bar{\delta}_{e}\right\}=\operatorname{res}_{K^{\prime}}(\operatorname{Inv} G(K))$ and Let $\overline{\boldsymbol{\sigma}}_{e+1}, \ldots, \overline{\boldsymbol{\sigma}}_{e+f}$ be a set of generators for $\mathscr{G}\left(K^{\prime} / K\right)$ such that $f \geq 2$. Let $\delta_{1}, \ldots, \delta_{e} \in \operatorname{Inv} G(K)$ extend $\bar{\delta}_{1}, \ldots, \bar{\delta}_{e}$, respectively. The set $S$ of all $(e+f)$-tuples $\left(\sigma_{1}, \ldots, \sigma_{e+f}\right) \in G(K)^{e+f}$ such that $\operatorname{res}_{K^{\prime}} \sigma_{l}=1$ for $i=1, \ldots, e$ and $\operatorname{res}_{K^{\prime}} \sigma_{l}=\bar{\sigma}_{t}$ for $i=e+1, \ldots, e+f$ is of positive measure (with respect to the normalized Haar measure of $G(K)^{e+f}$ ). Therefore by [5, Proposition 5.6], there exists an $(e+f)$-tuple $\left(\sigma_{1}, \ldots, \sigma_{e+f}\right) \in S$ such that, denoting $\varepsilon_{i}=\delta_{i}^{\sigma_{i}}$ for $i=1, \ldots, e$, we have: $K_{\sigma}=\tilde{K}\left(\varepsilon_{1}, \ldots, \varepsilon_{e}\right.$, $\left.\sigma_{e+1}, \ldots, \sigma_{e+f}\right)$ is a PRC field and

$$
G\left(K_{\sigma}\right)=\left\langle\varepsilon_{1}, \ldots, \varepsilon_{e}, \sigma_{e+1}, \ldots, \sigma_{e+f}\right\rangle \cong \hat{D}_{e, f} .
$$

In particular, $\operatorname{res}_{K^{\prime}}\left(G\left(K_{\sigma}\right)\right)=\mathscr{G}\left(K^{\prime} / K\right)$ and $\varepsilon_{1}, \ldots, \varepsilon_{e}$ represent the conjugacy classes of Inv $\mathrm{G}\left(K_{\sigma}\right)$, by Lemma 4.1(b). Hence

$$
\operatorname{res}_{K^{\prime}}\left(\operatorname{Inv} G\left(K_{\sigma}\right)\right)=\left\{\bar{\delta}_{1}, \ldots, \bar{\delta}_{e}\right\}=\operatorname{res}_{K^{\prime}}(\operatorname{Inv} G(K)) \supseteq \pi(\operatorname{Inv} G) .
$$

By Lemma 4.5, $K_{\sigma}$ has a Galois extension $N$ such that $K^{\prime} K_{\sigma} \cap N=$ $K_{\sigma}, G(N) \cong \hat{D}_{\omega}$ and $\operatorname{Inv} G(N)=\operatorname{Inv} G\left(K_{\sigma}\right)$. In particular $\operatorname{res}_{\mathrm{K}^{\prime}}(G(N))$ $=\mathscr{G}\left(K^{\prime} / K\right)$ and $\pi(\operatorname{Inv} G) \subseteq \operatorname{res}_{K^{\prime}}(\operatorname{Inv} G(N))$. It follows from Proposi-

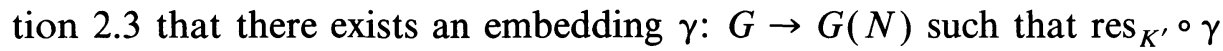
$=\pi$. The field $E=\tilde{K}(\gamma(G))$ is an algebraic extension of a PRC field $K_{\sigma}$, hence [11, Theorem 3.1] $E$ is PRC. Now $\gamma: G \rightarrow G(E)$ is an isomorphism and (1) commutes.

\section{REFERENCES}

[1] E. Binz, J. Neukirch and G. H. Wenzel, A subgroup theorem for free products of profinite groups, J. Algebra, 19 (1971), 104-109.

[2] T. Craven, The topological space of orderings of a rational function field, Duke Math. J., 41 (1974), 339-347.

[3] A. Douady, Cohomologie des groupes compacts totalement discontinus, Séminaire Bourbaki 1959-1960, exposé 189.

[4] J. Dugundji, Topology, Allyn and Bacon, Inc., Boston 1976. 
[5] D. Haran and M. Jarden, The absolute Galois group of a pseudo real closed field, to appear in Annali della Scuola Normale Superiore di Pisa.

[6] , Real free groups, to appear in J. Pure Appl. Algebra.

[7] J. Hocking and G. Young, Topology, Reading, Mass., Addison-Wesley, 1961.

[8] M. Jarden, An analogue of Čebotarev density theorem for fields of finite corank, J. Math., Kyoto University, 20 (1980), 141-147.

[9] M. Jarden and U. Kiehne, The elementary theory of algebraic fields of finite corank, Inventiones Math., 30 (1975), 275-294.

[10] A. Lubotzky and L. van den Dries, Subgroups of free profinite groups and large subfields of $\tilde{\mathbf{Q}}$, Israel J. Math., 39 (1980), 25-45.

[11] A. Prestel, Pseudo real closed fields, in: Set theory and model theory, 127-156, Lecture Notes in Mathematics 782, Springer, Berlin, 1981.

[12] L. Ribes, Introduction to profinite groups and Galois cohomology, Queen's University, Kingston, 1970.

Received December 11, 1984. The first author was supported by Alexander von Humboldt Foundation. The second author was partially supported by the fund for Basic Research administered by the Israel Academy of Sciences and Humanities.

DePaRTMENT OF MATHEMATICS

RUTGERS UNIVERSITY

NEW BRUNSWICK, NJ 08903

USA

AND

School of Mathematical Sciences

THE RAYMONd AND BEVERly SACKLER

FACUlTy OF Exact SCIENCES

Tel Aviv UnIVERSITY

Ramat Aviv Tel Aviv 69978

ISRAEL 



\section{PACIFIC JOURNAL OF MATHEMATICS EDITORS}

V. S. VARADARAJAN

(Managing Editor)

University of California

Los Angeles, CA 90024

HERBERT CLEMENS

University of Utah

Salt Lake City, UT 84112

R. FINN

Stanford University

Stanford, CA 94305
HERMANN FLASCHKA

University of Arizona

Tucson, AZ 85721

RAMESH A. GANGOLLI

University of Washington

Seattle, WA 98195

VAUGHAN F. R. JONES

University of California

Berkeley, CA 94720

ROBION KIRBY

University of California

Berkeley, CA 94720
C. C. MOORE

University of California

Berkeley, CA 94720

H. SAMELSON

Stanford University

Stanford, CA 94305

HAROLD STARK

University of California, San Diego

La Jolla, CA 92093

\section{ASSOCIATE EDITORS}

R. ARENS

E. F. BECKENBACH

B. H. NEUMANN (1906-1982)
F. WOLF

K. YOSHIDA

\section{SUPPORTING INSTITUTIONS}

UNIVERSITY OF ARIZONA

UNIVERSITY OF BRITISH COLUMBIA

CALIFORNIA INSTITUTE OF TECHNOLOGY

UNIVERSITY OF CALIFORNIA

MONTANA STATE UNIVERSITY

UNIVERSITY OF NEVADA, RENO

NEW MEXICO STATE UNIVERSITY

OREGON STATE UNIVERSITY
UNIVERSITY OF OREGON UNIVERSITY OF SOUTHERN CALIFORNIA

STANFORD UNIVERSITY

UNIVERSITY OF HAWAII

UNIVERSITY OF TOKYO

UNIVERSITY OF UTAH

WASHINGTON STATE UNIVERSITY

UNIVERSITY OF WASHINGTON 


\section{Pacific Journal of Mathematics}

\section{Vol. 123, No. $1 \quad$ March, 1986}

Maria Emilia Alonso García, A note on orderings on algebraic varieties $\ldots \ldots 1$

F. S. De Blasi and Józef Myjak, On continuous approximations for

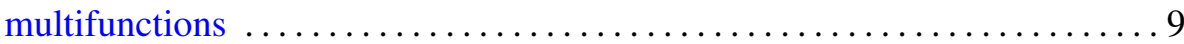

Frank Albert Farris, An intrinsic construction of Fefferman's CR metric . . . 33 Antonio Giambruno, P. Misso and Francisco César Polcino Milies, Derivations with invertible values in rings with involution $\ldots . \ldots . \ldots .47$

Dan Haran and Moshe Jarden, The absolute Galois group of a pseudo real

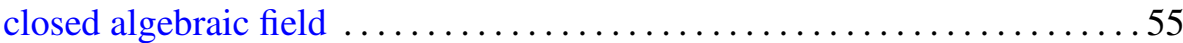

Telemachos E. Hatziafratis, Integral representation formulas on analytic

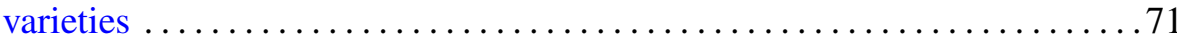

Douglas Austin Hensley, Dirichlet's theorem for the ring of polynomials

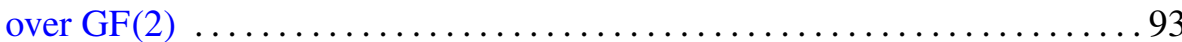

Sofia Kalpazidou, On a problem of Gauss-Kuzmin type for continued

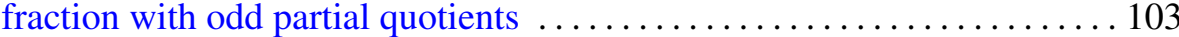

Harvey Bayard Keynes and Mahesh Nerurkar, Ergodicity in affine

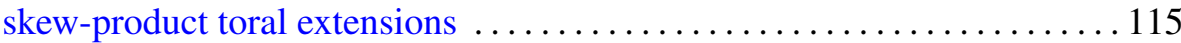

Thomas Landes, Normal structure and the sum-property $\ldots \ldots \ldots \ldots \ldots 127$

Anthony To-Ming Lau and Viktor Losert, Weak*-closed complemented invariant subspaces of $L_{\infty}(G)$ and amenable locally compact groups ...149 Andrew Lelek, Continua of constant distances in span theory . . . . . . . . 161 Dominikus Noll, Sums and products of $B_{r}$ spaces $\ldots \ldots \ldots \ldots \ldots \ldots \ldots \ldots$ Lucimar Nova, Fixed point theorems for some discontinuous operators 189

A. A. S. Perera and Donald Rayl Wilken, On extreme points and support points of the family of starlike functions of order $\alpha$

Massimo A. Picardello, Positive definite functions and $L^{p}$ convolution operators on amalgams ........................

Friedrich Roesler, Squarefree integers in nonlinear sequences ......... 223

Theodore Shifrin, The osculatory behavior of surfaces in $\mathbf{P}^{5}$ 